example because of cognitive, physical or emotional difficulties (Murphy et al., 2013; Murphy \& Boa, 2012; Murphy, 2009). Aims We aimed to introduce and use Talking Mats in a hospice setting and to develop a symbol set to support conversations relating to Advance Care Planning (ACP).

Methods Sixteen staff from the multidisciplinary team were trained to use Talking Mats and successfully used it with patients in the hospice. A sub-group of staff attended a workshop to discuss the potential use of Talking Mats to support conversations relating to ACP. Topics and options were agreed. These were then presented to a wider forum of staff from another Hospice for validation and checking. Three main topics to support ACP conversations were identified: Affairs; Care and Personal Values and are currently being trialled with a range of patients in the hospice setting.

Results Staff trained in the use of Talking Mats found that they could use it with a range of patients for a variety of purposes: getting to know someone; identifying goals; discharge planning and enabling ACP discussions.

Conclusion Talking Mats can be used by trained staff in a hospice setting to support people to express their views and help them plan for the end of life.

\section{P-13 IMPROVING ACP UPTAKE BY UNDERSTANDING AND ADDRESSING BARRIERS FACED BY HOSPICE STAFF}

Brenda Ward, Sarah Bache. John Taylor Hospice, Birmingham, UK

\subsection{6/bmispcare-2017-hospice.40}

Background ACP is a key means of improving care for people nearing the end of life. Enables better planning and provision of appropriate care (End of Life Care Programme, 2008). Although recognised as important, ACP conversations are not always carried out, confirmed by baseline audit (2015) of ACPs by patients with brain tumours. Results suggested some ACP occurring but scope to improve.

Methods Exploratory study to consider attitudes, enablers and possible barriers for hospice staff engaging in ACP. Need for good understanding of ACP and assistance with communication skills highlighted.

Following study, ACP Steering Group formed, actions included:

- ACP workshops for clinical staff.

- Review of an appropriate ACP paper document for patients to supplement oral information.

- New ACP template for hospice electronic records.

ACP workshops:

$5 \times 2$ hour, at hospice, attended by hospice MDT (March September, 2016). Consisted of presentation covering various aspects of ACP then time allowing staff opportunity to practice communication in role-play using 'fish bowl' technique.

Results Attendees:

Doctors (4), IPU nurses (9), CNS (community) (9) Pharmacy (4), Day Hospice (3), MDT (4) Administrator (1)

$100 \%$ attendees: workshop met training needs, most appreciated 'fish bowl' exercise some requested additional training most requested further opportunities to practice challenging conversations. Feedback on new electronic template: allows clear recording, easy to find recorded conversations, raises ACP profile. Follow up ACP audits: December 2016, April 2017. Results include 95\% patient records: evidence of complex discussions about ACP with hospice HCPs. Given the popularity of experiential training and recognising the need to assist staff with communication, further communication skills workshops arranged. Feedback: assist in increasing confidence and skills in addressing challenging conversations including ACP discussions.

Conclusion Hospice staff need training and support with ACP. Understanding and addressing needs in a variety of methods results in both an increase of ACP conversations and more confident staff.

\section{P-14 ADVANCE CARE PLANNING FACILITATOR}

Caitlyn Adkins. Kemp Hospice, Kidderminster, UK

\subsection{6/bmjspcare-2017-hospice.41}

Background It is recognised within the national framework that palliative and end of life (EoL) care must be a priority. Empowering individuals to think about their wishes and what is important to them is an extremely important and developing part of healthcare. The Advance Care Planning (ACP) Facilitator role was developed to support ACP within local care homes. After a three year project the role became permanent receiving full funding from the local Clinical Commissioning Group.

Aims The aim of the role is to support local care homes with ACP. Supporting them to achieve the national ambition that states everyone approaching EoL must be given the opportunity to plan. The role provides care home staff with support and education regarding EoL care. This allows them to work towards improving outcomes wherever the setting, which is a priority within the national framework.

Methods ACP support has been provided to care home staff and residents. Free educational sessions have been delivered on subjects relating to palliative and EoL care. Work has been undertaken within the local community to enhance their knowledge and understanding on ACP. A good working relationship has been developed with the multidisciplinary team to encourage a pro-active response to ACP.

Results Increased use of ACP documents has been noted within care homes. Good attendance and evaluations from the educational sessions have been recorded through registers and feedback forms. Verbal feedback has been received from numerous individuals with gratitude of the support provided.

Conclusion The role has shown to benefit residents, their loved ones and the staff. Residents are given the opportunity to discuss and record future plans which are in accordance with their wishes. Residents' loved ones are able to access various services the hospice offers. Care home staff have expressed feeling more confident with ACP and EoL care.

\section{P-15 THINKING AHEAD: COMPLEXITIES OF RESUSCITATION DECISIONS WITH DIVERSE COMMUNITIES IN LEICESTERSHIRE}

1,2Zoebia Islam, 1,2Lucy Taylor, 1,2 Helen Eborall, 1,2Christina Faull. 'LOROS, Leicester, UK; ${ }^{2}$ University of Leicester, Leicester, UK

10.1136/bmjspcare-2017-hospice.42

Background Advance care planning (ACP) supports people who are seriously ill to be cared for in the way, and in the place that they prefer. However, evidence suggests there are 
lower levels of ACP in people from Black, Asian and Minority Ethnic (BAME) communities. BAME groups are also more likely to desire invasive medical interventions, regardless of prognosis and impact on quality of life. Little is known about how the model of resuscitation decision-making fits with the social, cultural and religious values and beliefs of BAME groups. Evidence also suggests that health care professionals (HCPs) report a lack of confidence in having culturally appropriate discussions with BAME patients and their families. Equipping professionals to be more confident about such ACP discussions with BAME patients may lead to achieving patient preferences.

Aims With a focus on making decisions about resuscitation, this study explores professional views and experiences of ACP with patients from BAME backgrounds. It aims to identify barriers and enablers and person-centred outcomes to such discussions and provide evidence for training professionals.

Methods Thematic analysis of qualitative semi-structured interviews with HCPs across primary, secondary and tertiary care in Leicester, including GPs, hospital doctors and nurses.

Results There was an emphasis on the significance of building rapport, the timing of discussions and navigating communication barriers. Barriers to decision-making included: patients' and their families understanding of both prognosis and resuscitation; and differing values amongst generations of migrants. Professionals struggled with how to find a balance between acting in a non-discriminatory way whilst respecting cultural differences. Most HCPs highlight the need for further training and/or support.

Conclusion There are significant barriers for HCPs when discussing resuscitation decisions with people from BAME communities. This increases the complexity of navigating ACP and achieving patients' preferences. HCPs would benefit from further training and support.

\section{P-16 CONVERSATIONS ON LIVING AND DYING: FACILITATING ADVANCE CARE PLANNING FOR OLDER PEOPLE WITH FRAILTY}

${ }^{1}$ Sarah Combes, ${ }^{1,2}$ Caroline Nicholson, ${ }^{1}$ Karen Gillett, ${ }^{1}$ Christine Norton. ${ }^{1}$ King's College London, London, UK; ${ }^{2}$ St Christopher's Hospice, London, UK

\subsection{6/bmispcare-2017-hospice.43}

Background Older people living with frailty (older people) often receive suboptimal end-of-life care (Lloyd et al., 2016). Frequently under- or over-treated, older people experience inappropriate hospital admissions and procedures (Houben et al., 2014; Hunt et al., 2014). Most die in hospital despite $81 \%$ wishing to die at home (Office for National Statistics 2016). Advance care planning (ACP), a structured conversation between professionals and someone nearing the end-of-life, enables people to discuss and document what matters to them regarding their future care (Thomas \& Lobo, 2011) Facilitating informed decision-making regarding ACP means people are more likely to receive person-centred end-of-life care (National Council for Palliative Care, 2011). Multiple challenges mean that ACP is relatively uncommon for older people (Musa et al., 2015; Pollock \& Wilson, 2015). Consequently, priorities are often not discussed prior to significant deterioration (Sharp et al., 2013). leading to crisis decision-making which older people may not have capacity for (Clegg, 2013). Current initiatives encompass aspects of ACP.
However, there is no systematic, agreed format or training for health and social care professionals (professionals) that supports older people to articulate their broader end-of-life wishes on an ongoing basis (Brinkman-Stoppelenburg et al., 2014; Johnson et al., 2015; Weathers et al., 2016).

Aims This four-phase study aims to develop a relevant ACP intervention in collaboration with older people, their carers and professionals. Phase one, presented here, aims to establish current evidence regarding the components and implementation of ACP for community-dwelling older people, and use this to develop a theoretical model which will underpin the remaining study phases.

Methods An integrative review will be conducted using CINAHL, Embase, Ovid Medline, PsycINFO, and NHS Evidence databases, grey literature, and hand-searching. Paper selection will be verified by the research team and critically appraised using relevant systematic tools. Whittemore and Knafl's (2005) method will be used to develop an integrated, theoretical model. This will include data mapping against COM-B, a model of behaviour change often used in intervention development, to identify relevant target behaviours. (Michie et al., 2011)

Results and Conclusions The study began in January 2017. Phase one is currently underway and its results, conclusions and recommendations will be presented at this conference.

\section{\begin{tabular}{|l|l}
\hline P-17 WE DON'T TALK ANYMORE - IMPROVING \\
\hline
\end{tabular} COMMUNICATION OF ADVANCE CARE PLANNING ON DISCHARGE FROM HOSPITAL}

${ }^{1}$ Lorna Fairbairn, ${ }^{2}$ Dee Traue. ${ }^{1}$ Sue Ryder Manorlands Hospice, West Yorkshire, UK; ${ }^{2}$ East and North Herts NHS Trust, UK

10.1136/bmjspcare-2017-hospice.44

Background Research shows that more than 30\% of hospital inpatients over 85 may die within the next year. Advance Care Planning (ACP) has been shown to increase achievement of preferred place of death and decrease unnecessary hospital admissions in the last year of life. Accordingly, local Elderly Care discharge summaries include a mandatory ACP section. As part of the 'Building on the Best' quality improvement programme, our Trust is focusing on improving handover of ACP information as people move between healthcare settings.

Aims To determine best practice in sharing information on ACP between hospital and community services

To review current transfer of information about ACP on discharge from an Elderly Care ward.

Method We reviewed relevant literature about transfer of information on discharge summaries and retrospectively audited 30 discharges from an elderly care ward. We recorded inclusion of key ACP topics, such as cardiopulmonary resuscitation status and preferred place of death, as well as deaths within the subsequent six months.

Results Literature review highlighted importance of high quality information in discharge summaries to decrease inappropriate readmission in last year of life. No discharge summaries audited included any information in the 'mandatory' ACP section

$50 \%$ documented DNAR status separately

$53 \%$ of patients were readmitted to our hospital within six months

$30 \%$ died within six months. 